\title{
Nierenzellkarzinom: Sunitinib ist die am häufigsten eingesetzte Therapie
}

- Neue Daten aus dem deutschen Nierenkrebs-Register belegen den Status von Sunitinib (Sutent ${ }^{\circledR}$ ) und Temsirolimus (Torise ${ }^{\circledR}$ ) als Standardtherapien in der Erstlinienbehandlung des metastasierten Nierenzellkarzinoms (mRCC). Demnach ist Sunitinib mit einem Anteil von 62,7\% die am häufigsten eingesetzte mRCC-Therapie, gefolgt von Temsirolimus mit 11,8\%. „Eine robuste Datenlage und nachgewiesene Effektivität sind klare Kriterien für die Therapieentscheidung“, erläutert Privatdozent Dr. Axel Merseburger, Hannover. Wichtig seien darüber hinaus praktische Erfahrungen und Überlegungen zur Sequenz, die zurzeit die Diskussion prägen.

„Um die Tumorlast wirksam zu reduzieren und Resistenzen zu verhindern, sollte die effektivste Therapie am Anfang stehen“, betonte Merseburger. Da beim RCC ein Zusammenhang zwischen initialer Tumorlast und Überleben gezeigt wurde, kann durch die maximale Tumorschrumpfung auch ein Überlebensvorteil erreicht werden.
Die Evidenz zu Folgetherapien sei derzeit jedoch noch unklar, sodass weitere Therapieentscheidungen individuell getroffen werden müssten, so Dr. Merseburger. Es gebe aber erste Hinweise darauf, dass eine fortgesetzte VEGF-Hemmung vorteilhaft sein könnte.

Die mit Sunitinib erzielten Ansprech- und Überlebensdaten wurden bisher in keiner Phase-III-Studie mit einer anderen zielgerichteten Therapie bei mRCC gezeigt. In der Zulassungsstudie konnte das progressionsfreie Überleben unter dem MultikinaseInhibitor im Vergleich zu IFN- $\alpha$ verdoppelt und die objektive Ansprechrate vervierfacht werden. Das Gesamtüberleben betrug 26,4 Monate. Vieles spricht dafür, diese hohe Effektivität möglichst lange auszunutzen. Langzeitdaten deuten darauf hin, dass die Chance einer Remission unter Sunitinib mit zunehmender Therapiedauer steigt.

Nach Informationen von

Pfizer Oncology, Berlin

\section{Belastungsinkontinenz - Duloxetin als wichtiger Baustein der konservativen Therapie}

als auch in Kombination mit Beckenbodentraining einem alleinigen Beckenbodentraining oder keiner aktiven Therapie überlegen war.

Nach Erfahrungen aus der Praxis können ein Auftitrieren des Wirkstoffs sowie die gezielte Aufklärung der Patientin die Verträglichkeit der Therapie verbessern und somit auch die Compliance unterstützen. Für eine umfassende Behandlung der Patientinnen kann ein gut strukturiertes Patientinnenmanagement hilfreich sein. Wie so etwas aussehen kann, zeigt eine Online-Fortbildung, die das Unternehmen Lilly für die Betreuung belastungsinkontinenter Patientinnen entwickelt hat. Unter www. yentreve.de oder www.lillyfortbildung.de können Interessierte das Fortbildungsmodul herunterladen.

Nach Informationen von

Lilly, Bad Homburg
Online-Weiterbildung in der Uro-Onkologie

Das Internet-Portal www.cme-pcaarena.de bietet zertifizierte Fortbildung im Bereich der Uro-Onkologie. Das von Ferring unterstützte Portal umfasst unterschiedliche Module zu Diagnostik, Therapie und Grundlagenforschung. Diskutiert werden Themen wie die Suche nach diagnostischen Markern des Prostatakarzinoms oder neue Therapiemöglichkeiten wie adjuvante Strahlentherapie nach radikaler Prostatektomie. Die CME-Module sind mit jeweils drei Punkten von der Bayerischen Landesärztekammer zertifiziert.

Nach Informationen von Ferring, Kiel

\section{Einziges zugelassenes IPP-Medikament}

Für die medikamentöse Therapie der Induratio Penis Plastica (IPP) ist Potaba-Glenwood ${ }^{\circledR}$ mit dem Wirkstoff Kalium-4-Aminobenzoat das einzige zugelassene Präparat. Eine medikamentöse Behandlung kommt für Patienten in der frühen, instabilen Phase der Erkrankung infrage - als Interimslösung bis zur Stabilisierung der Progression und bei Patienten, die eine Operation nicht wünschen.

\section{Nach Informationen von} Glenwood, Starnberg

\section{Bärentraube bei Zystitis}

Ein Trockenextrakt aus Bärentraubenblättern (Cystinol akut ${ }^{\circledR}$ ) kann bei der Therapie einer akuten unkomplizierten Zystitis hilfreich sein. Ausschlaggebend ist der enthaltene Wirkstoff Arbutin. Arbutin ist zunächst inaktiv, wird im Körper zu Hydrochinonverbindungen umgewandelt und schließlich über den Urin ausgeschieden. Die E.coli-Bakterien im Urin spalten die Hydrochinonverbindungen zu einem aktiven Wirkstoff - dem freien Hydrochinon. Die Wirkweise von Cystinol akut ${ }^{\circledR}$ ist daher unabhängig vom $\mathrm{pH}$-Wert des Urins und macht somit eine begleitende Ernährungsumstellung nicht notwendig.

Nach Informationen von Schaper \& Brümmer, Salzgitter-Ringelheim 